\title{
Gender Disparity in the Relationship between Prevalence of Thyroid Nodules and Metabolic Syndrome Components: The SHDC-CDPC Community-Based Study
}

\author{
Xiaoying Ding, ${ }^{1,2}$ Ying Xu, ${ }^{3}$ Yufan Wang, ${ }^{1}$ Xiaohua Li, ${ }^{1}$ Chunhua Lu, ${ }^{4}$ Jing Su, ${ }^{1}$ \\ Yuting Chen, ${ }^{1}$ Yuhang Ma, ${ }_{1}^{1}$ Yanhua Yin, ${ }^{1}$ Yong $\mathrm{Wu},{ }^{3}$ Yaqiong Jin, ${ }^{3}$ Lihua Yu, ${ }^{4}$ Junyi Jiang, \\ Naisi Zhao, ${ }^{6}$ Qingwu Yan, ${ }^{2}$ Andrew S. Greenberg, ${ }^{2}$ Haiyan Sun, ${ }^{1}$ Mingyu Gu, ${ }^{1}$ Li Zhao, ${ }^{1}$ \\ Yunhong Huang, ${ }^{1}$ Yijie Wu, ${ }^{1}$ Chunxian Qian, ${ }^{3}$ and Yongde Peng' \\ ${ }^{1}$ Department of Endocrinology and Metabolism, Shanghai General Hospital, Shanghai Jiao Tong University School of Medicine, \\ Shanghai 200080, China \\ ${ }^{2}$ Jean Mayer USDA Human Nutrition Research Center on Aging, Tufts University, Boston, MA 02111, USA \\ ${ }^{3}$ Department of Internal Medicine, Sijing Hospital, Shanghai 201601, China \\ ${ }^{4}$ Department of Chronic Disease Prevention and Control, Sijing Community Health Service Centre of Songjiang District, \\ Shanghai 201601, China \\ ${ }^{5}$ Shanghai Pudong New Area Center for Disease Control and Prevention, Shanghai 200136, China \\ ${ }^{6}$ Department of Public Health and Community Medicine, Tufts University School of Medicine, Boston, MA 02111, USA
}

Correspondence should be addressed to Chunxian Qian; dxy_gh@sina.com and Yongde Peng; pengyongde0908@126.com

Received 22 October 2016; Accepted 20 February 2017; Published 21 May 2017

Academic Editor: Yong Wu

Copyright (C) 2017 Xiaoying Ding et al. This is an open access article distributed under the Creative Commons Attribution License, which permits unrestricted use, distribution, and reproduction in any medium, provided the original work is properly cited.

The study is aimed to investigate the pathogenesis underlying the increased prevalence of thyroid nodule (TN) in different levels of metabolic syndrome (MetS) components and analyze the relationships between TN and MetS components. A total of 6,798 subjects, including 2201 patients with TN, were enrolled in this study. Anthropometric, biochemical, thyroid ultrasonographic, and other metabolic parameters were all measured. There was obviously sexual difference in the prevalence of TN (males $26.0 \%$, females $38.5 \%$, resp.). The prevalence of TN in hyperuricemia (45.7\% versus $37.4 \%, P=0.001)$, NAFLD (41.2\% versus $36.4 \%, P<0.05$ ), and MetS (41.4\% versus $35.4 \%, P<0.001)$ groups was significantly increased only in females. Insulin resistance [OR $=1.31(1.15$, $1.49)]$, MetS $[\mathrm{OR}=1.18(1.03,1.35)]$, and diabetes $[\mathrm{OR}=1.25(1.06,1.48)]$ were all independent risk factors for TN in total subjects, whereas, after stratified analysis of gender, MetS $[\mathrm{OR}=1.29,(1.09,1.53)]$ and diabetes $[\mathrm{OR}=1.47,(1.17,1.84)]$ are still strongly and independently associated with the higher risks of TN in female subjects, but not in males. Our results suggest that the components of MetS might associate with the higher risks of TN in women than in men, but further cohort study of this gender disparity in the association between TN and MetS is required.

\section{Introduction}

Thyroid nodule (TN), one of the most common clinical thyroid diseases, has been becoming increasingly prevalent all over the world in the last decades and its associated risk factors have received much attention [1]. It is estimated that TN affects $4 \%$ to $7 \%$ of adults by palpation and $19 \%$ to $67 \%$ with ultrasonography [2], with 5 to $10 \%$ being malignant worldwide $[3,4]$. Thus, more thyroid nodule diagnoses mean more possibilities of the thyroid cancer occurrence in the future. Further study of the relevant risk factors of the TN is required.

Previous studies have showed that impaired glucose metabolism is an independent risk factor for increased thyroid volume and nodule prevalence [5-7]. Obesity was 
associated with higher risks of TN and thyroid cancer [810]. Insulin resistance (IR) was also shown to promote the formation and growth of TN [11]. Recently, it has been suggested that metabolic syndrome (MetS) was associated with the functional and morphological alterations of the thyroid gland and may be involved in the pathogenesis of TN $[12,13]$. Although the metabolic risk factors such as obesity, insulin resistance, and abnormal glucose metabolism are involved in the pathogenesis of TN in patients and these have been targeted for therapeutic intervention [14, 15], however, up to now, the metabolic mechanisms facilitating TN in individuals still have not been fully investigated, but also there has been scarce literature investigating the different levels of MetS risk factors in subjects with or without TN. Little is known about the relationships between TN and the components of MetS components [16], which limits the understanding of the mechanisms of the relative crosstalk between TN and MetS. TN are most frequently observed in females and in the elderly $[17,18]$; nevertheless, there is very little epidemiological data related to the gender disparity in the relationship between TN and the components of MetS in aged populations.

Based on this issue, the main purpose of this study was to investigate the prevalence of $\mathrm{TN}$ among a population aged over 45 years with different glucose metabolic status and to comprehensively investigate the association between TN diagnosed on ultrasonography and the MetS components in the SHDC-CDPC Community-based Study (Shenkang Hospital Development Center for Chronic Disease Prevention and Control project, Shanghai, China). A total of 7,920 individuals with age above 45 years were enrolled in the epidemiological investigation in a rural Chinese population. The different levels of metabolic indices between the TN group and control group were measured and compared. Our study would strengthen the associations between TN and the components of MetS and increase knowledge in gender disparity on the prevalence of $\mathrm{TN}$.

\section{Subjects and Methods}

2.1. Participants and Data Collection. From October 2014 to July 2015, a total of 7,920 local inhabitants aged 45 years or older who had been living in Sijing, Shanghai, for 1 year or longer before the enrollment and represented ten rural communities, were enrolled in this cross-section survey. A comprehensive survey was administered by the trained research staff to obtain a detailed questionnaire, anthropometry index, medical history, family histories of chronic diseases, and current medication use. Meanwhile, smoking and drinking status were also recorded. Through multiple screenings, 476 individuals were excluded from the study with missing data on questionnaire, anthropometry index, demographic variables, physical examination data, or the glucose metabolic indexes. Furthermore, subjects who met the exclusion criteria, including illnesses, such as hypothyroidism, hyperthyroidism, chronic renal failure, excessive drinking (an alcohol intake $>140 \mathrm{~g} /$ week for men or $>70 \mathrm{~g} /$ week for women), or current medication use affecting body composition, thyroid function, lipid profile, serum UA level, and glucose metabolic status, were excluded in the data analysis. In the end, a total of 6798 subjects and 2201 of them with TN were included in the final data analysis. The study protocol has been approved by the Committee on Human Research at Shanghai General Hospital, Shanghai Jiao Tong University School of Medicine. Written informed consent was obtained from each participant.

2.2. Anthropometric Measurement and Ultrasonography. All subjects had a physical examination in a fasting state. Blood pressure was measured in the all participants seated quietly for at least five minutes thrice consecutively and the average of three measurements was recorded. Waist circumference (WC) was measured in standing subjects, midway between the lower edge of the costal arch and the top of the iliac crest. Hip circumference (HC) was measured in standing subjects, around the widest portion of the buttocks. Body mass index (BMI) was calculated as body weight $/ \mathrm{height}^{2}$ in $\mathrm{kg} / \mathrm{m}^{2}$. Waistto-hip ratio (WHR) was calculated as WC divided by HC. In a supine position and the hyperextended neck of all participants, ultrasound examination of the thyroid nodules, including the TN number and location, was performed and evaluated independently by the two senior experts using a B-mode high-resolution tomographic ultrasound system (Toshiba, Tokyo, Japan).

\subsection{Biochemical Measurements and Calculation. Venous} blood samples were collected from all participants in the morning after an overnight fasting for at least 10 hours. The subjects without diagnosis of diabetes underwent the oral standard $75 \mathrm{~g}$ glucose tolerance test (OGTT) and the previously diagnosed diabetes underwent the steamed bread meal test. Biochemical measurements, including plasma glucose concentrations, uric acid (UA), serum lipid profile containing levels of total cholesterol (TCH), low-density lipoprotein cholesterol (LDL-C), high-density lipoprotein cholesterol (HDL-C), and triglycerides (TG), were measured enzymatically using an automatic biochemistry analyzer (HITACHI 7600). Fasting plasma insulin (FINS) concentration, serum level of thyroid-stimulating hormone (TSH), free triiodothyronine (FT3) concentration, free tetraiodothyronine (FT4) concentration, and thyroid peroxidase antibody (TPOAB) concentration were measured by electrochemiluminescence analyzer (Roche Diagnostics, Basel, Switzerland). The homoeostasis model assessment for insulin resistance index (HOMA-IR) was calculated by multiplying fasting plasma insulin (mIU/l) and fasting plasma glucose (FPG) $(\mathrm{mM})$ and dividing the result by 22.5 . Beta cell function (HOMA-beta) was calculated as 20x fasting plasma insulin $(\mathrm{mIU} / \mathrm{l}) /(\mathrm{FPG}(\mathrm{mM})-3.5) \times 100 \%$. Glycosylated hemoglobin (HbAlc) was measured by high-performance liquid chromatography (D10; Bio-Rad Laboratories, Inc., CA).

2.4. Definition and Diagnostic Criteria. The MetS was defined according to the IDF criteria [19] with modification on WC cutoff point for an Asian population: the central obesity (defined as WC $\geq 90 \mathrm{~cm}$ for men or $\geq 80 \mathrm{~cm}$ for women; [20]), 
plus any two or more: (1) raised TG $(\geq 1.7 \mathrm{mmol} / \mathrm{l}$ or specific treatment for this lipid abnormality); (2) reduced HDL-C $(<1.03 \mathrm{mmol} / \mathrm{l}$ in men and $<1.29 \mathrm{mmol} / \mathrm{l}$ in women or specific treatment for this lipid abnormality); (3) raised blood pressure $(\geq 130 / 85 \mathrm{mmHg}$ or treatment of previously diagnosed hypertension); (4) raised fasting plasma glucose ( $\geq 5.6 \mathrm{mmol} / \mathrm{l}$ or previously diagnosed type 2 diabetes). The diabetes and prediabetes were defined using criteria recommended by the ADA 2010 [21]. BMI $\geq 25 \mathrm{~kg} / \mathrm{m}^{2}$ was defined as overweight or $\geq 30 \mathrm{~kg} / \mathrm{m}^{2}$ was defined as obesity using criteria recommended by the World Health Organization [22]. Insulin resistance was evaluated using HOMA-IR of 2.8 or higher [23]. Hyperuricemia was defined as serum uric acid level of $420 \mathrm{umol} / \mathrm{L}$ or higher in men and $360 \mathrm{umol} / \mathrm{L}$ or higher in women, respectively [24]. Current smoking status (Yes or No) was defined according to smoking more than one cigarette daily for at least 6 months. Current alcohol consumption status (Yes or No) was defined according to drink more than one time of any type monthly. A thyroid nodule is a discrete lesion within the thyroid gland that is radiologically distinct from the surrounding thyroid parenchyma [25]. NAFLD was defined according to the "Diagnostic Criteria of Nonalcoholic Fatty Liver Disease by the Chinese Society of Hepatology" after exclusion of viral or autoimmune liver disease and excessive alcohol consumption [26]. NAFLD was ascertained using hepatic ultrasonography that revealed ultrasound beam attenuation, a diffusely increased echogenicity in the liver parenchyma or poor visualization of intrahepatic structures by two trained ultrasonographists [27].

2.5. Statistical Analysis. All statistical analyses were performed using the SAS version 9.2 (SAS Institute Inc., Cary, NC, USA). Demographic, metabolic features and other clinical parameters were described by sex using frequency (percentage) for categorical variables and median (interquartile range) for continuous variables, respectively. Additionally, we divided the participants into different subgroups with and without thyroid nodules according to the different levels of MetS components and clinical characteristics. Differences on metabolic characteristics in subjects with or without TN were evaluated using $\chi^{2}$ test for the categorical variables or using nonparameter Wilcoxon test for analysis for the continuous variables. To further explore whether metabolic syndrome is associated with the risk of TN, unconditional sex-stratified logistic regression models were used to estimate the adjusted odds ratios (ORs) and 95\% confident intervals (CIs) of MetS components with TN prevalence. Significance tests were twotailed, with $P$ value less than 0.05 considered of statistical significance.

\section{Results}

3.1. General Characteristics of Subjects with or without Thyroid Nodules. Clinical characteristics of the total of 6,798 participants including 3289 males and 3509 females, with a median age of 58.8 years (52.5-66.0), stratified by gender with and without thyroid nodules, were presented in Table 1. Of the 6798 subjects, the prevalence of TN was $32.4 \%$. The prevalence of TN in women was significantly higher $(38.5 \%)$ than in men $(26.0 \%)(P<0.001)$. Regardless of gender, analysis of the clinical risk factors revealed that subjects with TN were significantly older and had higher levels of SBP, FPG, PPG, HbAlc, FINS, HOMA-IR, HOMA-beta, FT4, and TPOAB than subjects without TN. Furthermore, all subjects with TN had significantly lower levels of DBP, FT3, and TSH than those without TN. However, after further gender stratification, there were still significant differences of SBP, UA, FPG, PPG, FINS, HOMA-IR, and TSH between the two groups in females.

\subsection{Stratified Analysis of Prevalence of Thyroid Nodules} according to the Different Metabolic Status. To explore the association of $\mathrm{TN}$ and related metabolic risk factors, the subjects were classified into different subgroups according to the different levels of MetS components and clinical characteristics (Table 2). The prevalence of TN was significantly increasing in the elder subjects $(P$ for trend $<0.001)$ and insulin resistance group $(31.0 \%$ in the total control group, $37.0 \%$ in the total IR group, $P<0.001 ; 24.9 \%$ in the male control group, $30.1 \%$ in the male IR group, $P<0.001$; $37.2 \%$ in the female control group, $42.0 \%$ in the female IR group, $P<0.01$ ), respectively. However, there were no significant differences in the prevalence of TN between the male and female subgroups with the different levels of body fat accumulation (divided by BMI; by WHR quartile; by waist circumference) except for higher prevalence of TN in total subjects with central obesity. The prevalence of TN with normal glucose metabolism, prediabetes, and diabetes was $29.9 \%, 31.4 \%$, and $37.2 \%$, respectively, whereas the significant differences were only in female subjects $(33.3 \%, 37.9 \%$, and $46.0 \%$, resp., $P<0.001)$. To explore whether MetS and the associated other metabolic parameters contributed to the pathogenesis of $\mathrm{TN}$, all subjects were further divided into different subgroups according to being with or without MetS, with or without NAFLD, and with or without hyperuricemia, respectively. The results showed that the prevalence of TN with hyperuricemia ( $45.7 \%$ versus $37.4 \%, P=0.001$ ), NAFLD (41.2\% versus $36.4 \%, P<0.05)$, and MetS (41.4\% versus $35.4 \%, P<0.001)$ groups was statistically significantly higher in females, but not in males.

3.3. Logistic Regression Analyses of the Associated Metabolic Risk Factors of TN in Male and Female Subjects. Finally, to explore whether the MetS and the associated other metabolic parameters were independently associated with TN. A multiple logistic regression analysis for the risk factors of TN involving all the significant different anthropometric and metabolic parameters, such as central obesity, HOMA-IR, HOMA-beta, FPG, PPG, FINS, hyperuricemia, NAFLD, and MetS, were applied in subjects with or without TN (Figure 1). We next performed the stratified analysis in the subgroups divided according to the serum UA levels, glucose metabolic status, HOMA-IR, FINS, thyroid function, waist circumference, NAFLD, and MetS. Analysis of logistic regression indicated that diabetes $[\mathrm{OR}=1.254,(1.061,1.481)]$, insulin resistance $[\mathrm{OR}=1.309,(1.149,1.490)]$, and MetS $[\mathrm{OR}=1.178$, 


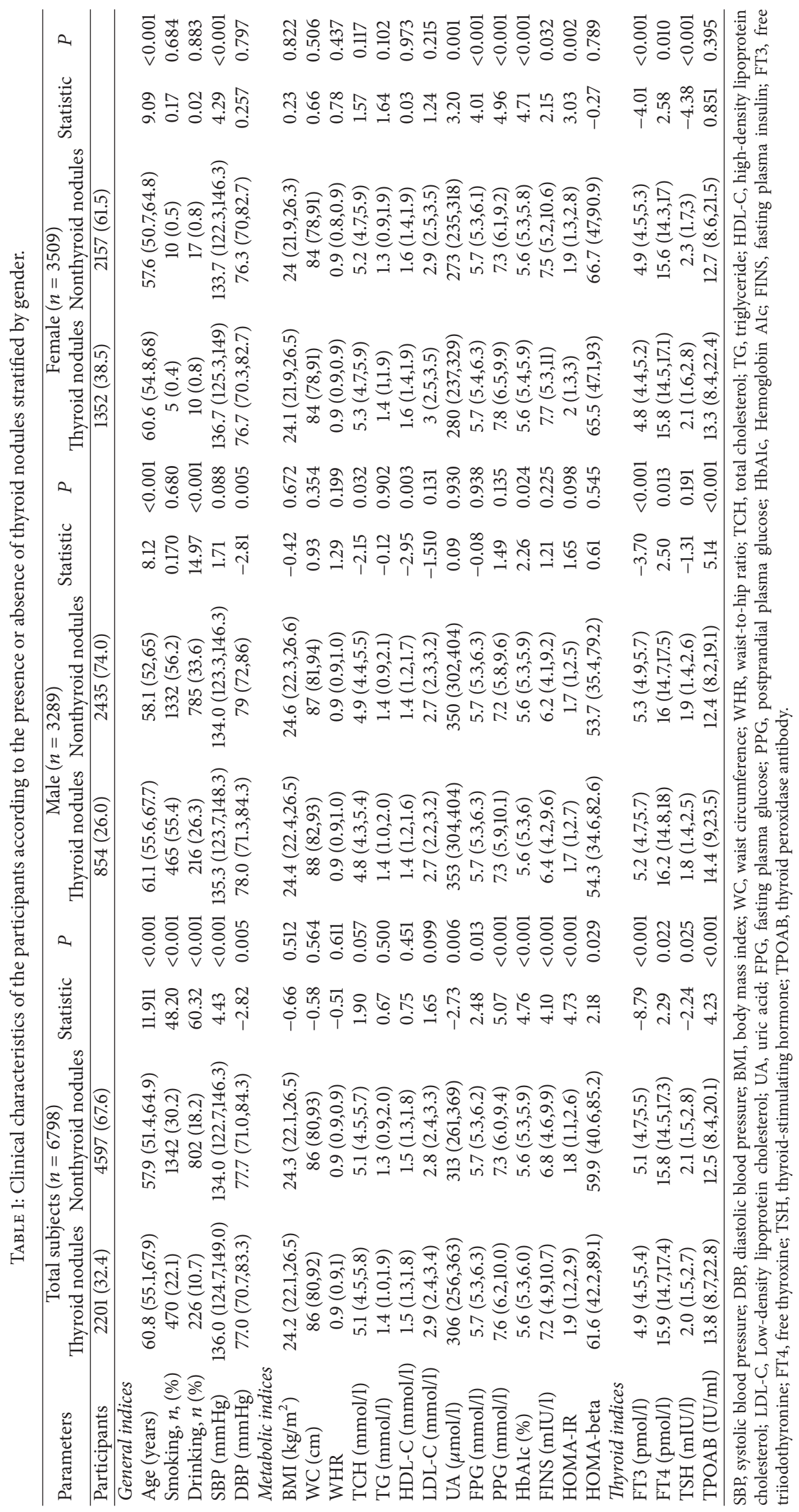




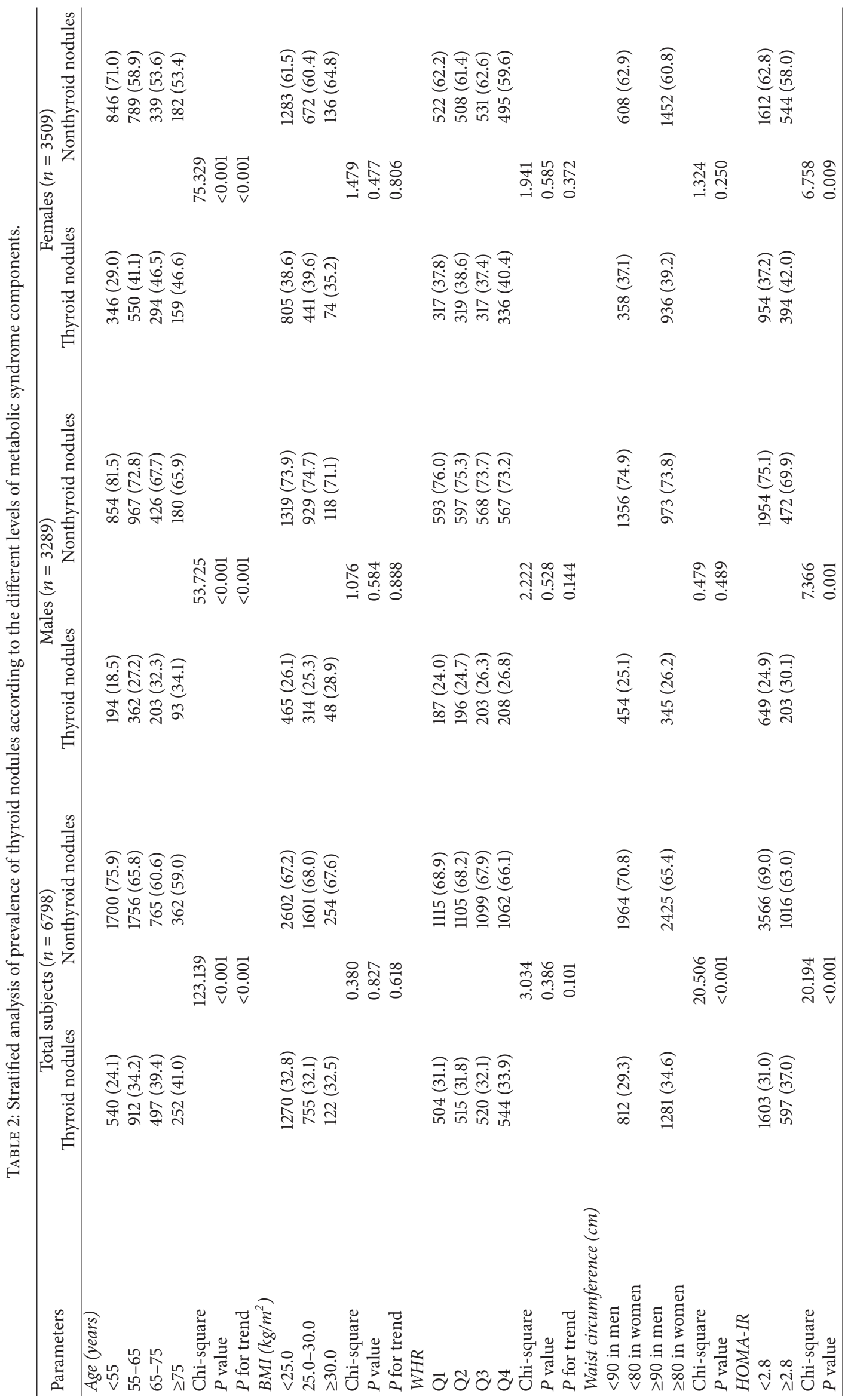




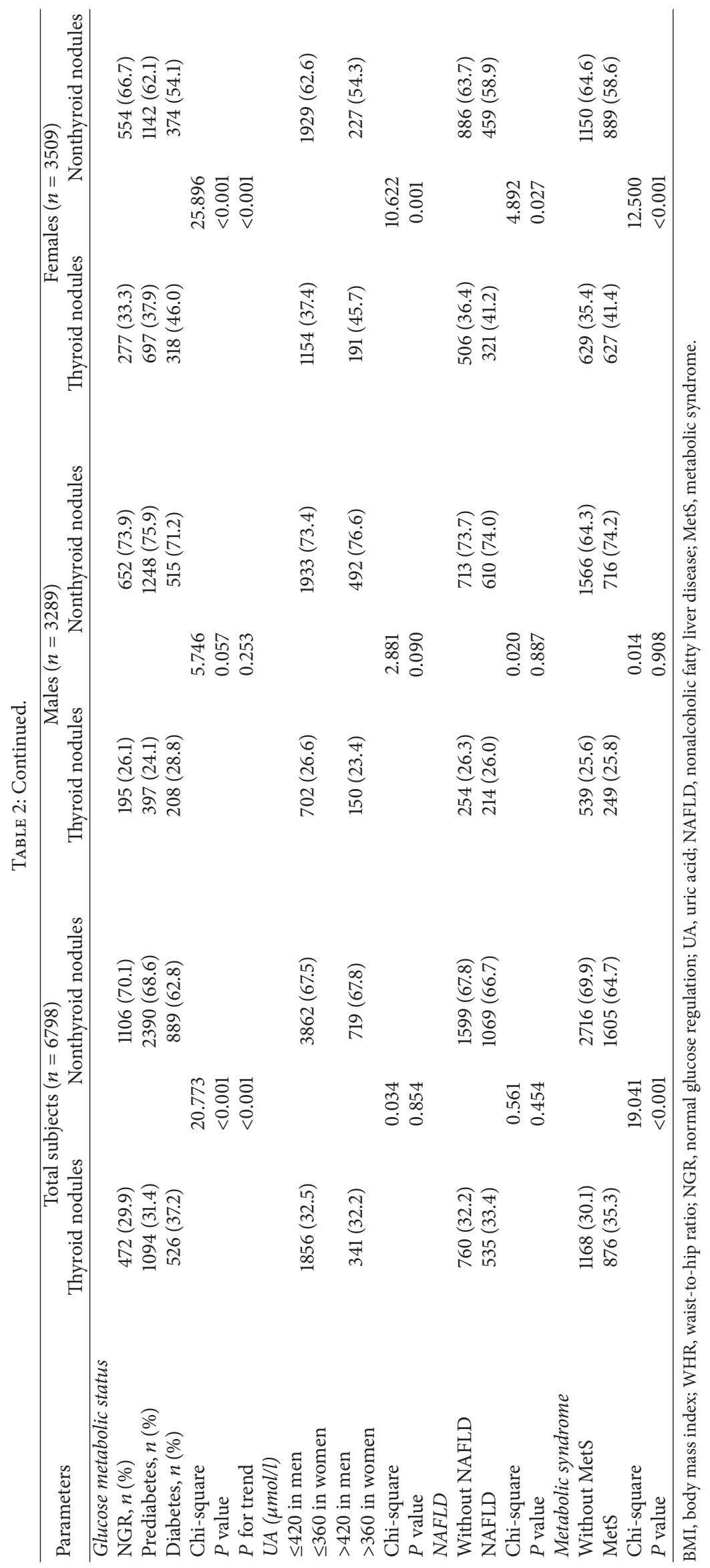




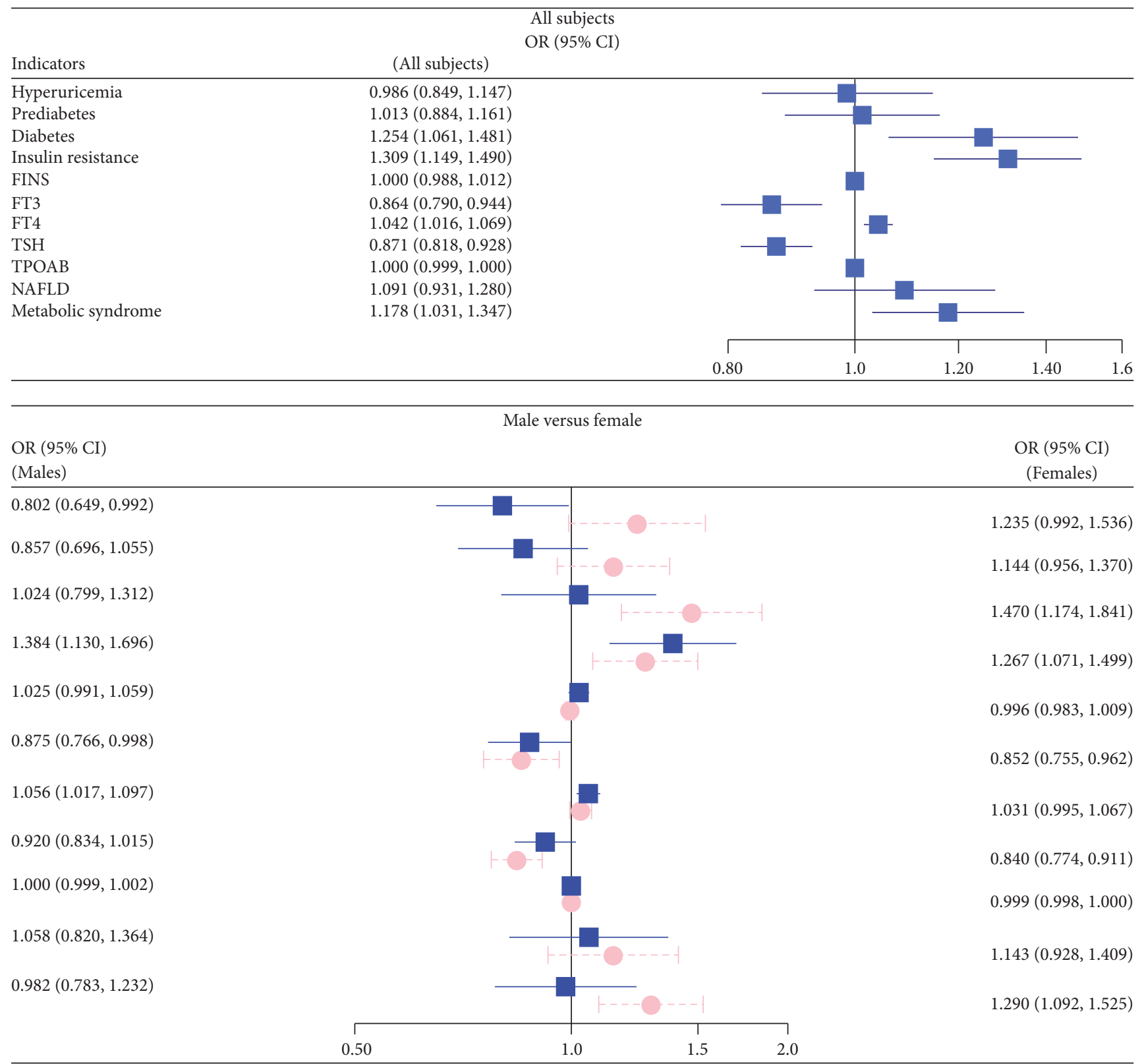

Males

Females

Figure 1: Metabolic risk factors of thyroid nodules were analyzed using logistic regression in total, male and female subjects. Adjustment of age, drinking, smoking, and family history of thyroid disease; FINS, fasting plasma insulin; FT3, free triiodothyronine; FT4, free thyroxine; TSH, thyroid-stimulating hormone; TPOAB, thyroid peroxidase antibody; NAFLD, nonalcoholic fatty liver disease.

(1.031, 1.347)], but neither hyperuricemia nor FINS, were all independently significant risk factors for the increased prevalence of TN in all subjects after additional adjusting for age, smoking, drinking status, and family history of thyroid disease. Additionally, the most pronounced sex disparity was found in the relationship between the prevalence of thyroid nodules and metabolic syndrome. After stratified analysis of gender, MetS $[\mathrm{OR}=1.29,(1.092,1.525)]$ and diabetes $[\mathrm{OR}=$
$1.47,(1.174,1.841)]$ are still strongly and independently associated with the higher risks of TN in female subjects, but not in males (Figure 1).

\section{Discussion}

The prevalence of TN and the accompanying thyroid tumors are the increasing public health problems [1, 28, 29]. 
Guo et al. [30] reported the population aged over 40 years had a higher prevalence $(46.6 \%)$ of TN previously. Our crosssectional study was performed in a large community-based population in rural China. Among men and women aged over 45 years, the prevalence of TN was one-third higher in women than in men (38.5\% versus $26 \%$, resp.). This frequency was higher with advancing age, among females and in subjects with insulin resistance. But, up to now, the mechanisms of the higher prevalence of TN in females as compared to the males are not completely understood. So we investigated the prevalence of TN in different levels of MetS components and examined whether MetS components and the associated metabolic risk factors contributed to the pathogenesis of TN in male and female subjects, respectively. Our results were partially comparable to previous studies, suggesting that females and the elderly were all risk factors for TN $[18,31,32]$. Moreover, we found that diabetes and MetS were independent risk factors for TN after adjusting for age, smoking, and alcohol consumption only in female subjects, but not in males.

Metabolic syndrome is a complex clinical disorder characterized by dyslipidemia, obesity, NAFLD, insulin resistance, hyperuricemia, and a disturbance of glucose metabolism. In our study, to further explore whether MetS components and the other metabolic risk parameters related to the pathogenesis of TN in subjects, all subjects were divided into different subgroups according to different metabolic status. Nearly $35.3 \%$ of subjects with MetS had TN. The results showed that the subjects with TN had significantly higher levels of FPG, PPG, HbAlc, FINS, HOMA-IR, HOMAbeta, and TPOAB than those without TN after adjusting for age. However, in addition to the above findings, after further stratified analysis of gender, there were still significant differences of FPG, PPG, FINS, HOMA-IR, and TSH between the groups with or without TN in females, but not in males. The females with hyperuricemia, NAFLD, or MetS had much higher prevalence of TN as compared with controls, whereas there were no such associations in males. Perhaps the most intriguing finding of our study was that the MetS were significantly associated with TN only in women. The one plausible explanation for this gender disparity in TN formation was the hormone testosterone, which might contribute to the protective roles against the harmful effects of MetS cluster in men compared to women. We concluded from our data that the prevalence of TN was more closely associated with the components of MetS in women than in men. Female subjects with MetS were at increased risk for TN. Few studies assessed gender disparity in the pathogenesis of TN formation [33]. The gender dichotomy of MetS-induced TN formation may underlie the increased propensity to $\mathrm{TN}$ in women. The gender differences observed in this study contributed to an increased theoretical understanding of TN in MetS and might suggest future studies into the sex-specific pathophysiology of TN in MetS, which remains to be further determined. Clinically, awareness of gender differences in the relationship between thyroid nodules and the components of MetS might help to detect TN in women with MetS. Therefore, better intervention strategies against the components of MetS might be performed to reduce the risks of TN occurrence.
Researchers have recently focused their interest on the pathogenesis of $\mathrm{TN}$ in subjects with abnormal glucose metabolism [6, 34]; however, the mechanisms facilitating TN in individuals with impaired glucose metabolism have not been investigated thoroughly [5]. In previous studies, strong correlations between thyroid volume, BMI, and WC were demonstrated $[8,16,35-37]$. Because the fat deposition in abdomen was linked to IR and MetS, the prevalence of TN in patients with central obesity or IR, which were the high risk states for development of diabetes, were significantly higher than that of controls in total subjects in our study. Ayturk et al. [38] discovered that IR was an independent risk factor for TN formation in iodine-sufficient areas, but the pathophysiologic mechanisms for the increased risk were still not fully understood. It has been reported that metformin which could improve IR might result in a significant decrease in the nodular size in patients with IR [39]. Nonetheless, there was limited data about the interaction between insulin resistance and the pathogenesis of TN $[11,40]$. In this study, the prevalence of $\mathrm{TN}$ in diabetes group was $37.2 \%$, which was significantly higher than $31.4 \%$ in the prediabetes and $29.9 \%$ in normal glucose tolerance (NGR). IR as an important metabolic factor in the development of the diabetes may have had an impact on the incidence of TN. We also found the risks of prevalence of $\mathrm{TN}$ were increased by 1.384 times in male and 1.267 times in female, with IR, respectively. It has been indicated that insulin receptors were overexpressed in most thyroid tumors. All these findings suggested the level of IR was the key factor most independently and strongly correlated with TN in all subjects. After stratified analysis of gender, MetS and diabetes were still strongly and independently associated with the higher risks of TN in female subjects, but not in males. These results indicated that there is a need for better understanding of gender disparity in the relationship between prevalence of TN and MetS components. Additional studies are required to discern how these findings may impact future research, diagnosis, and treatment of TN.

Recently, extensive studies have found the roles of NAFLD and hyperuricemia in IR. Up to now, we were unable to find any published studies to explore the role of NAFLD in pathogenesis of TN in a large population [41, 42]. The literature about the relationships between NAFLD, hyperuricemia, and TN remains scant. Concerns have been given to the relationship between hyperuricemia, inflammation, and IR recently. Since others have shown positive correlations between the levels of UA and IR [40], we inferred that the hyperuricemia may lead to the formation of thyroid nodules. The results of our study also showed that the prevalence of TN with hyperuricemia and NAFLD groups was statistically significantly higher in females. It has previously been reported that NAFLD and hyperuricemia, which by themselves are risk factors for the activation of inflammation pathways, share many predisposing metabolic risk factors with IR $[43,44]$. We tentatively put forward that IR, hyperuricemia, NAFLD, and MetS might play the important roles in TN formation. Therefore, in female subjects with IR, NAFLD, hyperuricemia, or MetS, more attention should be paid to the early and the timely medical management. 
Ayturk et al. reported that higher serum TSH level was an independent risk factor for increased thyroid volume in MetS patients but failed to find the relationship between TSH and TN formation [38]. In our study, regarding free thyroid hormones, results appeared contradictory at TN prevalence. Higher FT3 and serum thyroid-stimulating hormone levels were related to a decreased risk of $\mathrm{TN}$ in female subjects, while higher FT4 level was related to an increased risk of TN in male subjects. A recent study reported that FT3 and FT3/FT4 were positively related to BMI, waist, TG, and FPG, while FT4 was negatively related to the above metabolic parameters [45]. Few studies have explored this apparent paradox between the free THs (thyroid hormones) and TN. Taken together, although the difference of the study populations might be responsible for the above inconsistency, it was still difficult to explain the independent association between TN formation and the free THs levels. A possible explanation could be that free T3 and free T4 might be affecting the prevalence of MetS in opposite ways [45-47]. In addition, our study should be acknowledged with several potential limitations. This study was cross-sectional in design and therefore no causal inferences can be drawn. Although we found different levels of SBP, UA, HOMA-IR, FT3, and FT4 as well as plasm glucose between subjects with or without TN, it was still hard to get any causal relationships. Furthermore, the findings of single-centre study could not have been used to all TN population regarding urbanization, economic development, and geographic distribution. Given these findings, prospective studies on a larger scale are required to clarify the causal associations of MetS, IR, and hyperuricemia with TN in subjects. Therefore, the early integrated intervention involving uric acid-lowering, lipid-regulating therapy, and insulin-sensitizing medication might more effectively delay the formation of TN than either intervention alone in women with MetS.

\section{Conclusion}

Although the components of metabolic syndrome lie among the risk factors for TN both in men and women, our results suggested that MetS components had the much stronger effects on the risk of TN in women than in men. In conclusion, age, gender, IR, MetS, and abnormal glucose metabolic status as well as hyperuricemia independently played the important roles in the pathological mechanisms of thyroid nodules. The prevalence of TN in female patients with MetS was significantly increased, which was significantly associated with the different levels of MetS components. The prevalence of TN in DM group was significantly higher than those in NGR and prediabetes groups only in females. The right managements of MetS aimed to adjust hyperuricemia, central obesity, abnormal glucose metabolism, and IR might be beneficial in the blocking of TN formation, especially in females. Our data supported the possible metabolic clues to the gender disparity of nodule formation. Hence, in the future, more cross-sectional and long-term cohort multicenter study on a large scale will be necessary to further verify and clarify the findings in this study.

\author{
Abbreviations \\ WHR: Waist-to-hip ratio \\ SBP: $\quad$ Systolic blood pressure \\ DBP: Diastolic blood pressure \\ BMI: Body mass index \\ WC: Waist circumference \\ HC: $\quad$ Hip circumference \\ NAFLD: Nonalcoholic fatty liver disease \\ TCH: Total cholesterol \\ TG: $\quad$ Triglyceride \\ HDL-C: High-density lipoprotein cholesterol \\ LDL-C: Low-density lipoprotein cholesterol \\ UA: $\quad$ Uric acid \\ FPG: $\quad$ Fasting plasma glucose \\ PPG: Postprandial plasma glucose \\ HbAlc: Hemoglobin Alc \\ FINS: Fasting plasma insulin \\ NGR: Normal glucose regulation \\ NAFLD: Nonalcoholic fatty liver disease \\ MetS: Metabolic syndrome \\ FT3: $\quad$ Free triiodothyronine \\ FT4: Free thyroxine \\ TSH: Thyroid-stimulating hormone \\ TPOAB: Thyroid peroxidase antibody.
}

\section{Conflicts of Interest}

The authors declare that there are no conflicts of interest.

\section{Authors' Contributions}

Xiaoying Ding, Ying Xu, and Yufan Wang contributed equally to this work.

\section{Acknowledgments}

The study was supported by grants from the Shanghai Shenkang Hospital Development Center for chronic disease prevention and control project (SHDC12015304), the Shanghai municipal health bureau key project fund (201440033), 2015 Wang Kuancheng medicine fund, 2015 Shanghai General Hospital Excellent physician project, the Songjiang district health bureau medical climbing project (0702N14003), and The science and technology committee project of Songjiang district (15SJGG54). The authors thank all the study participants and all the research staff for their participation and contribution.

\section{References}

[1] G. Pellegriti, F. Frasca, C. Regalbuto, S. Squatrito, and R. Vigneri, "Worldwide increasing incidence of thyroid cancer: update on epidemiology and risk factors," Journal of Cancer Epidemiology, vol. 2013, Article ID 965212, 10 pages, 2013.

[2] L.-Z. He, T.-S. Zeng, L. Pu, S.-X. Pan, W.-F. Xia, and L.-L. Chen, "Thyroid hormones, autoantibodies, ultrasonography, and clinical parameters for predicting thyroid cancer," International 
Journal of Endocrinology, vol. 2016, Article ID 8215834, 11 pages, 2016.

[3] I. S. Nam-Goong, H. Y. Kim, G. Gong et al., "Ultrasonographyguided fine-needle aspiration of thyroid incidentaloma: correlation with pathological findings," Clinical Endocrinology, vol. 60, no. 1, pp. 21-28, 2004.

[4] E. Papini, R. Guglielmi, A. Bianchini et al., "Risk of malignancy in nonpalpable thyroid nodules: predictive value of ultrasound and color-doppler features," Journal of Clinical Endocrinology and Metabolism, vol. 87, no. 5, pp. 1941-1946, 2002.

[5] C. Anil, A. Akkurt, S. Ayturk, A. Kut, and A. Gursoy, "Impaired glucose metabolism is a risk factor for increased thyroid volume and nodule prevalence in a mild-to-moderate iodine deficient area," Metabolism: Clinical and Experimental, vol. 62, no. 7, pp. 970-975, 2013.

[6] Y. Yeo, S. H. Ma, Y. Hwang et al., "Diabetes mellitus and risk of thyroid cancer: a meta-analysis," PLoS ONE, vol. 9, no. 6, Article ID e98135, 2014.

[7] A. O. Duran, C. Anil, A. Gursoy et al., "Thyroid volume in patients with glucose metabolism disorders," Arquivos Brasileiros de Endocrinologia e Metabologia, vol. 58, no. 8, pp. 824-827, 2014.

[8] A. Arduc, B. A. Dogan, M. M. Tuna et al., "Higher body mass index and larger waist circumference may be predictors of thyroid carcinoma in patients with Hürthle-cell lesion/neoplasm fine-needle aspiration diagnosis," Clinical Endocrinology, vol. 83, no. 3, pp. 405-411, 2015.

[9] W. Xu, Z. Chen, and N. Li, "Relationship of anthropometric measurements to thyroid nodules in a Chinese population," BMJ Open, vol. 5, no. 12, Article ID e008452, 2015.

[10] P. A. Sousa, M. Vaisman, J. R. Carneiro et al., "Prevalence of goiter and thyroid nodular disease in patients with class III obesity," Arq Bras Endocrinol Metabol, vol. 57, no. 2, pp. 120-125, 2013.

[11] Z. Heidari, M. A. Mashhadi, and S. Nosratzehi, "Insulin resistance in patients with benign thyroid nodules," Archives of Iranian Medicine, vol. 18, no. 9, pp. 572-576, 2015.

[12] J. Shin, M.-H. Kim, K.-H. Yoon, M.-I. Kang, B.-Y. Cha, and D.-J. Lim, "Relationship between metabolic syndrome and thyroid nodules in healthy Koreans," Korean Journal of Internal Medicine, vol. 31, no. 1, pp. 98-105, 2016.

[13] E. Blanc, C. Ponce, D. Brodschi et al., "Association between worse metabolic control and increased thyroid volume and nodular disease in elderly adults with metabolic syndrome," Metabolic Syndrome and Related Disorders, vol.13, no. 5, pp. 221226, 2015.

[14] C. Anil, A. Kut, B. Atesagaoglu, A. Nar, N. B. Tutuncu, and A. Gursoy, "Metformin decreases thyroid volume and nodule size in subjects with insulin resistance: A Preliminary Study," Medical Principles and Practice, vol. 25, no. 3, pp. 233-236, 2016.

[15] M. Karimifar, A. Aminorroaya, M. Amini et al., "Effect of metformin on thyroid stimulating hormone and thyroid volume in patients with prediabetes: a randomized placebo-controlled clinical trial," Journal of Research in Medical Sciences, vol. 19, no. 11, pp. 1019-1026, 2014.

[16] J. Yin, C. Wang, Q. Shao et al., "Relationship between the prevalence of thyroid nodules and metabolic syndrome in the iodine-adequate area of Hangzhou, China: a cross-sectional and cohort study," International Journal of Endocrinology, vol. 2014, Article ID 675796, 7 pages, 2014.

[17] H. Jiang, Y. Tian, W. Yan et al., "The prevalence of thyroid nodules and an analysis of related lifestyle factors in Beijing communities," International Journal of Environmental Research and Public Health, vol. 13, no. 4, article no. 442, 2016.

[18] N. Kwong, M. Medici, T. E. Angell et al., "The influence of patient age on thyroid nodule formation, multinodularity, and thyroid cancer risk," Journal of Clinical Endocrinology and Metabolism, vol. 100, no. 12, pp. 4434-4440, 2015.

[19] K. G. M. M. Alberti, P. Zimmet, and J. Shaw, "The metabolic syndrome-a new worldwide definition," The Lancet, vol. 366, no. 9491, pp. 1059-1062, 2005.

[20] Y. Xu, L. Wang, and J. He, "Prevalence and control of diabetes in Chinese adults," JAMA, vol. 310, no. 9, pp. 948-959, 2013.

[21] American Diabetes Association, "Diagnosis and classification of diabetes mellitus," Diabetes Care, vol. 34, supplement 1, pp. S62-S69, 2011.

[22] WHO, "Obesity: preventing and managing the global epidemic. Report of a WHO consultation,” World Health Organization Technical Report Series 894, 2000.

[23] W. Jia, "Status of insulin resistance in Chinese population," International Journal of Endocrinology and Metabolism, vol. 22, no. 4, p. 264, 2002.

[24] D. I. Feig, D.-H. Kang, and R. J. Johnson, "Medical progress: uric acid and cardiovascular risk," New England Journal of Medicine, vol. 359, no. 17, pp. 1811-1821, 2008.

[25] D. S. Cooper, G. M. Doherty, B. R. Haugen et al., "Revised American thyroid association management guidelines for patients with thyroid nodules and differentiated thyroid cancer," Thyroid, vol. 19, no. 11, pp. 1167-1214, 2009.

[26] F. Jian-gao, "Guidelines for management of nonalcoholic fatty liver disease: an updated and revised edition," Chinese Journal of Hepatology, vol. 18, no. 3, pp. 163-166, 2010.

[27] M. D. Zeng, J. G. Fan, L. G. Lu et al., "Guidelines for the diagnosis and treatment of nonalcoholic fatty liver diseases," Journal of Digestive Diseases, vol. 9, no. 2, pp. 108-112, 2008.

[28] S. R. Bomeli, S. O. LeBeau, and R. L. Ferris, "Evaluation of a thyroid nodule," Otolaryngologic Clinics of North America, vol. 43, no. 2, pp. 229-238, 2010.

[29] L. G. T. Morris, A. G. Sikora, T. D. Tosteson, and L. Davies, “The increasing incidence of thyroid cancer: the influence of access to care," Thyroid, vol. 23, no. 7, pp. 885-891, 2013.

[30] H. Guo, M. Sun, W. He et al., "The prevalence of thyroid nodules and its relationship with metabolic parameters in a Chinese community-based population aged over 40 years," Endocrine, vol. 45, no. 2, pp. 230-235, 2014.

[31] K. Kiseljak-Vassiliades and M. Xing, "Association of cigarette smoking with aberrant methylation of the tumor suppressor gene RAR $\beta 2$ in papillary thyroid cancer," Frontiers in Endocrinology, vol. 2, article 99, 2011.

[32] Y. S. Balhara and K. Deb, "Impact of alcohol use on thyroid function," Indian Journal of Endocrinology and Metabolism, vol. 17, no. 4, pp. 580-587, 2013.

[33] L. J. Bessey, N. B. K. Lai, N. E. Coorough, H. Chen, and R. S. Sippel, "The incidence of thyroid cancer by fine needle aspiration varies by age and gender," Journal of Surgical Research, vol. 184, no. 2, pp. 761-765, 2013.

[34] B. Aschebrook-Kilfoy, M. M. Sabra, A. Brenner et al., "Diabetes and thyroid cancer risk in the national institutes of HealthAARP diet and health study," Thyroid, vol. 21, no. 9, pp. 957-963, 2011.

[35] S. Semiz, U. Şenol, O. Bircan, S. Gümüşlü, S. Bilmen, and I. Bircan, "Correlation between age, body size and thyroid volume in an endemic area," Journal of Endocrinological Investigation, vol. 24, no. 8, pp. 559-563, 2001. 
[36] L. Hegedus, H. Perrild, L. R. Poulsen et al., "The determination of thyroid volume by ultrasound and its relationship to body weight, age, and sex in normal subjects," Journal of Clinical Endocrinology and Metabolism, vol. 56, no. 2, pp. 260-263, 1983.

[37] R. Sari, M. K. Balci, H. Altunbas, and U. Karayalcin, “The effect of body weight and weight loss on thyroid volume and function in obese women," Clinical Endocrinology, vol. 59, no. 2, pp. 258262, 2003.

[38] S. Ayturk, A. Gursoy, A. Kut, C. Anil, A. Nar, and N. B. Tutuncu, "Metabolic syndrome and its components are associated with increased thyroid volume and nodule prevalence in a mild-to-moderate iodine-deficient area," European Journal of Endocrinology, vol. 161, no. 4, pp. 599-605, 2009.

[39] J. Rezzónico, M. Rezzónico, E. Pusiol, F. Pitoia, and H. Niepomniszcze, "Metformin treatment for small benign thyroid nodules in patients with insulin resistance," Metabolic Syndrome and Related Disorders, vol. 9, no. 1, pp. 69-75, 2011.

[40] J. N. Rezzónico, M. Rezzónico, E. Pusiol, F. Pitoia, and H. Niepomniszcze, "Increased prevalence of insulin resistance in patients with differentiated thyroid carcinoma," Metabolic Syndrome and Related Disorders, vol. 7, no. 4, pp. 375-380, 2009.

[41] T. Geach, "Thyroid function: poor thyroid function linked to NAFLD," Nature Reviews Endocrinology, vol. 12, no. 8, p. 434, 2016.

[42] Y. Nakade and M. Yoneda, "Relationship between non-alcoholic fatty liver disease and thyroid dysfunction," Internal Medicine, vol. 55, no. 15, pp. 1941-1942, 2016.

[43] F. Martinon, "Update on biology: uric acid and the activation of immune and inflammatory cells," Current Rheumatology Reports, vol. 12, no. 2, pp. 135-141, 2010.

[44] S. Becker, L. Dossus, and R. Kaaks, "Obesity related hyperinsulinaemia and hyperglycaemia and cancer development," Archives of Physiology and Biochemistry, vol. 115, no. 2, pp. 86-96, 2009.

[45] S. Jing, D. Xiaoying, X. Ying et al., "Different levels of thyroid hormones between impaired fasting glucose and impaired glucose tolerance: free T3 affects the prevalence of impaired fasting glucose and impaired glucose tolerance in opposite ways," Clinical Endocrinology, vol. 80, no. 6, pp. 890-898, 2014.

[46] Y. Ye, X. Gai, H. Xie, L. Jiao, and S. Zhang, "Association between serum free thyroxine (FT4) and uric acid levels in populations without overt thyroid dysfunction," Annals of Clinical and Laboratory Science, vol. 45, no. 1, pp. 49-53, 2015.

[47] G. L. Roef, E. R. Rietzschel, C. M. Van Daele et al., “Triiodothyronine and free thyroxine levels are differentially associated with metabolic profile and adiposity-related cardiovascular risk markers in euthyroid middle-aged subjects," Thyroid, vol. 24, no. 2, pp. 223-231, 2014. 


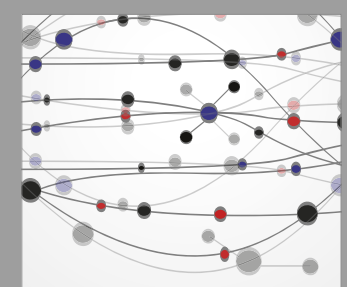

The Scientific World Journal
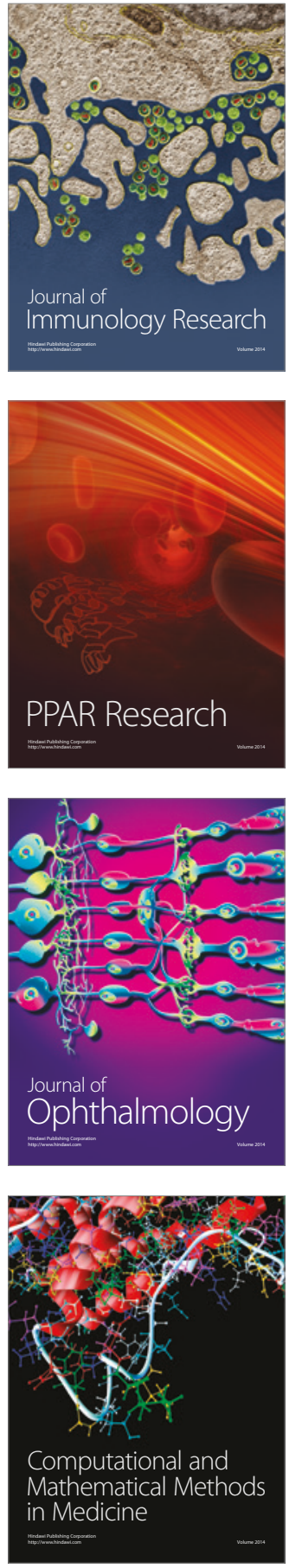

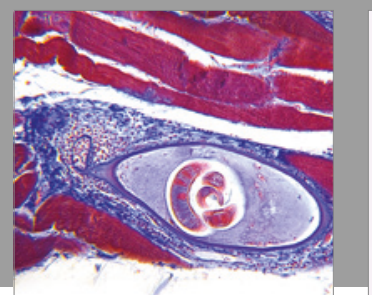

Gastroenterology Research and Practice
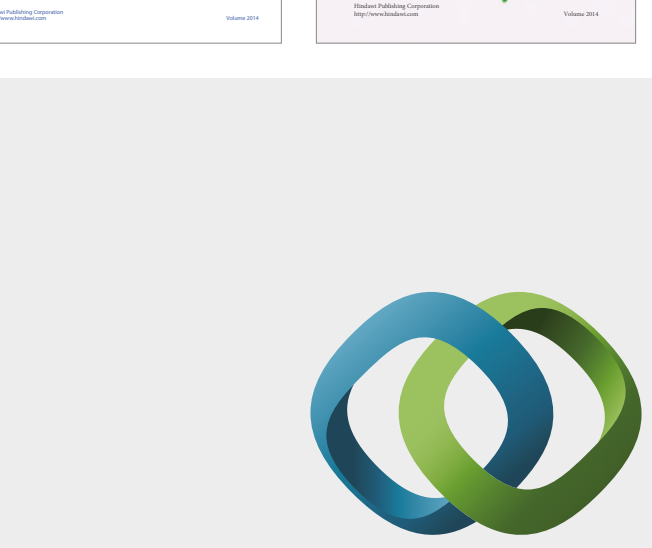

\section{Hindawi}

Submit your manuscripts at

https://www.hindawi.com
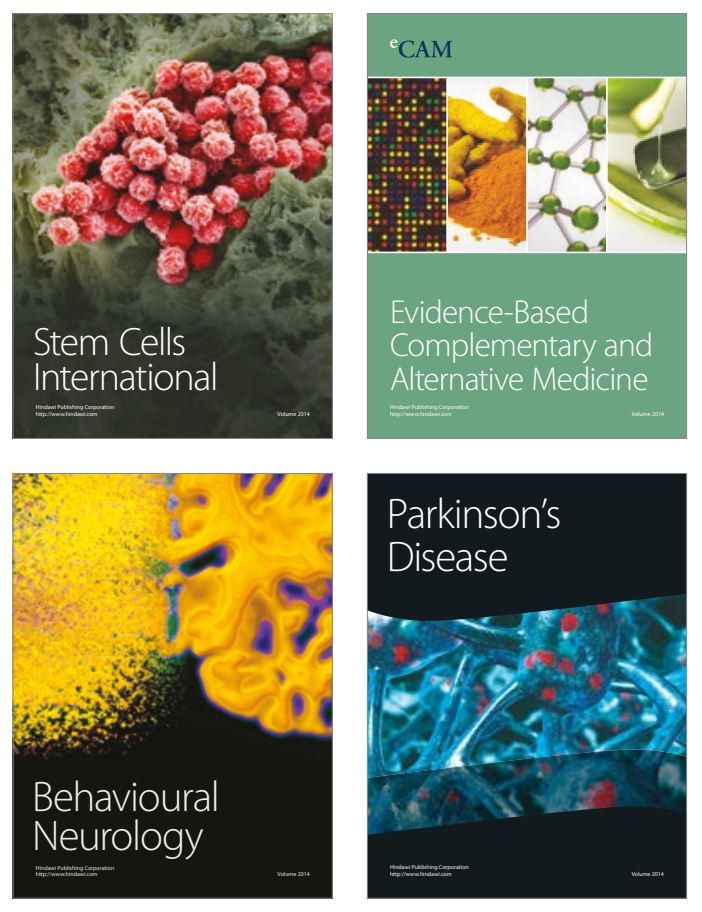
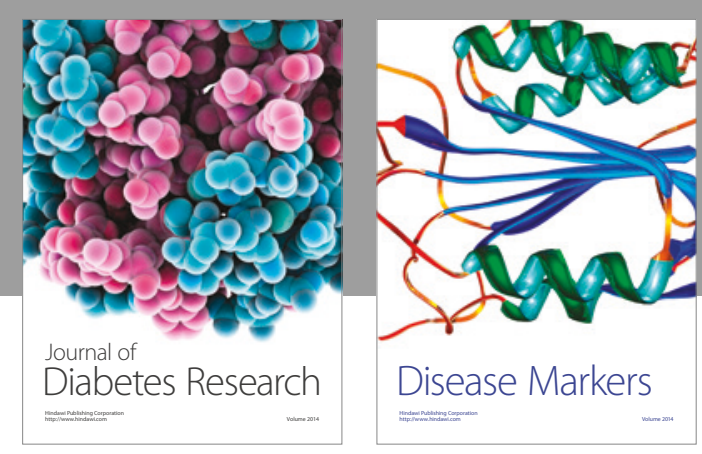

Disease Markers
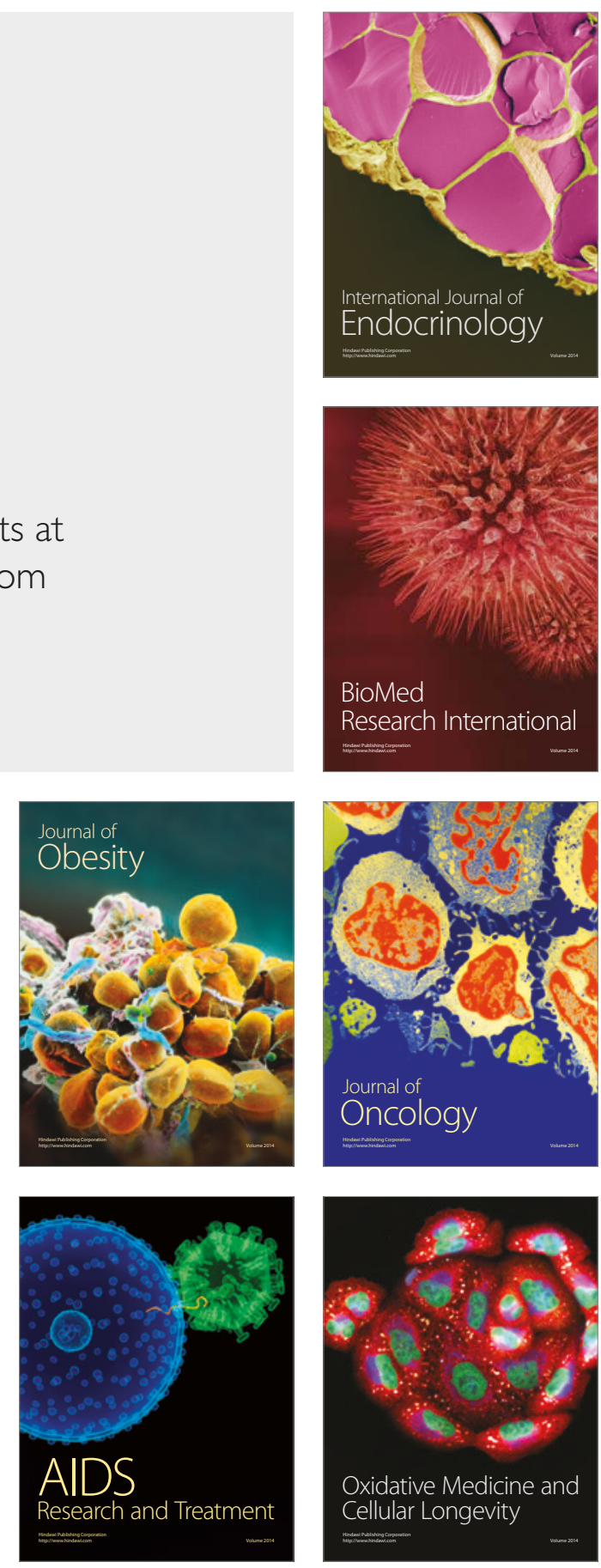